\title{
Costo-efectividad del tratamiento con interferón beta en pacientes con síndrome clínico aislado de alto riesgo en Colombia
}

\author{
Luis Alfonso Zarco¹, Sonia Patricia Millán¹, Darío Londoño², Ludy Parada³, Alejandra Taborda³, \\ Miguel Germán Borda ${ }^{4}$ \\ 1 Departamento de Neurociencias, Hospital Universitario San Ignacio, Facultad de Medicina, Pontificia \\ Universidad Javeriana, Bogotá, D.C., Colombia \\ 2 Unidad de Neumología, Hospital Universitario San Ignacio, Facultad de Medicina, Pontificia Universidad \\ Javeriana, Bogotá, D.C., Colombia \\ 3 Departamento de Epidemiología Clínica y Bioestadística, Facultad de Medicina, Pontificia Universidad Javeriana, \\ Bogotá, D.C., Colombia \\ 4 Facultad de Medicina, Pontificia Universidad Javeriana, Bogotá, D.C., Colombia \\ Estudio realizado en el Hospital Universitario San Ignacio. Bogotá, Colombia.
}

\begin{abstract}
Introducción. En 85 \% de los pacientes con esclerosis múltiple se presenta como manifestación inicial un primer evento desmielinizante o síndrome clínico aislado. En estos casos, el tratamiento con interferón beta retrasa hasta dos años la progresión a esclerosis múltiple. Sin embargo, en Colombia este medicamento es costoso.

Objetivo. Determinar si el tratamiento del síndrome clínico aislado con interferón beta es costo-efectivo al retrasar la esclerosis múltiple en dos años.

Materiales y métodos. Se realizó un análisis de costo-efectividad empleando un árbol de decisiones basado en la perspectiva del paciente y la sociedad. A partir de una revisión sistemática de la literatura y de conceptos de expertos se definieron las diversas probabilidades. Los costos de la enfermedad se calcularon por medio de la revisión de historias y la aplicación de encuestas a los pacientes atendidos en el Hospital Universitario San Ignacio. Para controlar la incertidumbre se realizó un análisis de sensibilidad mediante una simulación de Monte Carlo con mil pacientes.

Resultados. El costo del tratamiento con interferón sobrepasa los Col\$95'000.000 (US\$ 50.000) por paciente durante los dos años. Aproximadamente, $80 \%$ corresponde a los costos del medicamento. El costo de la recaída se acerca a Col\$39'139.200 (US\$21.744), y los costos indirectos corresponden a Col\$10'958.400 (US\$ 6.088). La tasa representativa del mercado fue de Col\$1.800. Con el tratamiento se ganan sólo 0,06 años de vida ajustados por discapacidad (AVAD) adicionales. La razón de costoefectividad 'incremental' (sic.) supera el umbral, incluso en el análisis de sensibilidad.

Conclusión. La administración de interferón beta en pacientes con síndrome clínico aislado de alto riesgo en los primeros dos años no es costo-efectiva en Colombia.
\end{abstract}

Palabras clave: esclerosis múltiple, interferón beta, análisis costo-beneficio, enfermedades desmielinizantes.

doi: http://dx.doi.org/10.7705/biomedica.v34i1.1541

The cost-effectiveness of interferon beta treatment in patients with a clinically isolated syndrome in Colombia

Introduction: Approximately $85 \%$ of patients with multiple sclerosis have an initial demyelinating event. Treatment with interferon beta delays the progression of multiple sclerosis for nearly two years in patients with a clinically isolated syndrome. In Colombia, interferon is very expensive when compared to other countries.

Objective: We sought to determine the cost-effectiveness of a two-year interferon beta treatment within Colombia in patients with a clinically isolated syndrome.

\footnotetext{
Contribución de los autores:

Luis Zarco y Sonia Millán: revisión sistemática de la literatura, preparación de manuscritos para aprobación del Comité de Ética.

Luis Zarco: asesoría clínica y convocatoria a grupo de expertos en enfermedades desmielinizantes.

Sonia Millán: revisión de historias clínicas, creación de bases de datos de los pacientes, aplicación de encuestas a pacientes con diagnóstico de esclerosis múltiple o síndrome clínico aislado para calcular los costos indirectos de la enfermedad.

Darío Londoño: diseño de asesoría y análisis epidemiológico y diseño de árbol de decisiones.

Ludy Parada y Alejandra Taborda: análisis de costo-utilidad y costo-efectividad bajo perspectiva del paciente y la sociedad.

Germán Borda: aplicación de encuestas a pacientes con diagnóstico de esclerosis múltiple o síndrome clínico aislado para calcular los costos indirectos de la enfermedad.
} 


\begin{abstract}
Materials and methods: Based on patient and society perspectives, a cost-effectiveness analysis was conducted using a decision tree. A variety of probabilities were defined after a systematic review of the available literature. The disease costs were calculated by reviewing medical charts at the Hospital San Ignacio University and surveys completed by multiple sclerosis patients. To control for uncertainty in these data, analysis of approximately one-thousand patients was performed using Monte Carlo methods.

Results: The two-year treatment cost per patient exceeds Col\$95,000,000 (US $\$ 50,000$ ). Approximately $80 \%$ of this cost corresponds to medications (US $\$ 40,500$ ). The price of relapse and indirect costs totals Col\$ 41,632,149 (US\$21,744) and Col\$ 11,656,389 (US\$ 6,088), respectively. Treatment represents an increase of 0.06 quality-adjusted life years (QALY). The incremental cost-effectiveness ratio exceeds the threshold, regardless of the use of Monte Carlo methods for analysis.

Conclusion: Administering interferon beta over the course of two years to high-risk patients with a clinically isolated syndrome is not cost-effective within Colombia.
\end{abstract}

Key words: multiple sclerosis, interferon-beta, cost-benefit analysis, demyelinating diseases. doi: http://dx.doi.org/10.7705/biomedica.v34i1.1541

La esclerosis múltiple es una enfermedad crónica con graves consecuencias a largo plazo, especialmente en el tema de discapacidad (1). Se estima que, aproximadamente, $85 \%$ de los pacientes con esta enfermedad presentan como manifestación inicial un primer evento desmielinizante o síndrome clínico aislado. De 30 a $70 \%$ de los pacientes con un primer evento clínico desmielinizante progresan a esclerosis múltiple (2-6).

La prevalencia de esclerosis múltiple en Colombia se ha estimado en 4,41 por 100.000 habitantes y, aunque es baja, su variedad clínica se distribuye principalmente en recaída-remisión (75,7%) (7).

Diversos estudios han demostrado que el uso del interferón en pacientes con síndrome clínico aislado retrasa la progresión de la enfermedad entre uno y dos años, disminuye la carga de la lesión en resonancia y la discapacidad (8-10). Se define síndrome clínico aislado como un episodio agudo o subagudo de alteración neurológica secundaria a una lesión focal en la sustancia blanca. Clínicamente, esta entidad se puede presentar como neuritis óptica (21\%), compromiso del tallo cerebral (10\%), afección de vías largas (46I \%) o síntomas multifocales $(231 \%)(5,6,10)$.

Probablemente, el factor más importante en el riesgo de progresión a esclerosis múltiple es la presencia de lesiones asintomáticas en la resonancia magnética cerebral $(11,12)$. Sin embargo, otros factores que se han asociado con la progresión a

\footnotetext{
Correspondencia:

Luis Alfonso Zarco, Departamento de Neurociencias, Hospital Universitario San Ignacio, Facultad de Medicina, Pontificia Universidad Javeriana, Carrera 7 N ${ }^{\circ}$ 40-62, Bogotá, D.C., Colombia

Teléfono: (571) 5946171 lazarco@javeriana.edu.co

Recibido: 15/01/13; aceptado: 09/10/13
}

esclerosis múltiple son la presencia de síndrome clínico aislado en adultos jóvenes y mujeres o la presencia de neuritis óptica unilateral $(3,13-19)$.

En términos generales, se considera que el tiempo promedio entre el síndrome clínico aislado y la aparición de un segundo episodio es de 1,7 años (20). En el estudio CHAMPS, $82 \%$ de los pacientes desarrollaron, por lo menos, una nueva lesión hiperintensa mayor de $10 \mathrm{~mm}$ en la secuencia T2 luego de 18 meses de seguimiento. Asimismo, en el estudio ETOMS, $94 \%$ de los pacientes con síndrome clínico aislado tenía, por lo menos, una lesión mayor de $10 \mathrm{~mm}$ y múltiples lesiones menores de $10 \mathrm{~mm}$ luego de dos años (21). En el BENEFIT, $85 \%$ de los pacientes del grupo placebo cumplía con los criterios de diagnóstico de esclerosis múltiple luego de dos años del primer evento $(1,2,20,22)$.

Existen diferentes estudios, como el CHAMPS, CHAMPIONS, ETOMS, BENEFIT y, más recientemente, el REFLEX, que evalúan el impacto del interferón beta en la progresión a esclerosis múltiple $(1-3,5,6,20,21,23)$. Con base en estos estudios, se ha podido concluir que el tratamiento con agentes modificadores de la enfermedad retrasa la progresión a esclerosis múltiple entre dos y tres años (24); igualmente, se ha concluido que la terapia con interferón es efectiva siempre y cuando se inicie tempranamente y que, por el contrario, su eficacia se ve limitada en pacientes con enfermedad avanzada $(9,10,15,25-27)$.

Se han desarrollado diferentes estudios que buscan evaluar los costos de la esclerosis múltiple (2830). Dichos estudios evalúan los costos derivados de la atención médica, las hospitalizaciones, las incapacidades y la discapacidad medida en la escala EDSS (Expanded Disability Status Scale). Según algunos resultados europeos, se 
estima que el costo anual de la enfermedad es de aproximadamente $€ 20.000$ para aquellos pacientes con escala de discapacidad de 4 y se incrementa a $€ 50.000$ cuando la escala es de 6,5. Cuando la escala puntúa 7 o más, los costos derivados de esta entidad oscilan entre $€ 60.000$ y 110.000 (8). Se estima que $11 \%$ del total de costos corresponde a los agentes modificadores de la enfermedad $(8,31)$. Los análisis de costos se desarrollaron a partir del análisis de la información obtenida de las tarifas hospitalarias, costos de medicamentos, incapacidades y pérdidas de productividad (8).

Hay estudios con interferón beta1A que sugieren que la administración temprana de este fármaco es costo-efectiva $(14,15,26,32)$; sin embargo, los resultados presentan variaciones importantes. En general, aunque significativo, el beneficio que reportan los estudios es marginal y el costo del medicamento es alto. Por todo lo anterior, este trabajo es de alto impacto en nuestro medio.

Se realizó un estudio cuyo objetivo fue determinar si el tratamiento temprano con interferón beta en pacientes con síndrome clínico aislado de tipo I es costo-efectivo para disminuir la progresión a esclerosis múltiple definitiva en dos años.

\section{Materiales y métodos}

Mediante un árbol de decisiones se realizó un análisis de costo-efectividad con un horizonte temporal corto de dos años (figura 1). La efectividad del interferón beta en pacientes con síndrome clínico aislado de alto riesgo se determinó a partir de una revisión sistemática de la literatura por medio de una búsqueda realizada entre septiembre de 2010 y noviembre de 2011 en las bases de datos Medline, MD-Consult, Ovid, Proquest, Ebsco, Lilacs, Hinari y ScienceDirect con los siguientes criterios de búsqueda: clinically isolated syndrome, interferon beta, multiple sclerosis y cost-effectiveness.

La población se seleccionó con base en las indicaciones existentes para el inicio del tratamiento con interferón beta. El tiempo de seguimiento de dos años se tomó con base en los resultados de estudios, como el BENEFIT, que demostraron beneficio en retrasar la aparición de la enfermedad por este lapso. Los datos de la progresión a esclerosis múltiple se tomaron de los estudios CHAMPS, ETOMS y BENEFIT $(10,17,21)$ (cuadro 1).

Con el objeto de calcular los costos de la intervención y del proceso de la enfermedad se revisaron las historias clínicas de los pacientes que habían consultado al Hospital Universitario San Ignacio entre febrero de 2009 y septiembre de 2011 con diagnóstico de esclerosis múltiple o síndrome clínico aislado (cuadro 2).

Los costos del interferón se calcularon con base en el valor máximo de recobro de unidad por tratamiento mensual de interferón beta $1 \mathrm{~b}$ señalado en la Resolución 0005 de 2011 del Ministerio

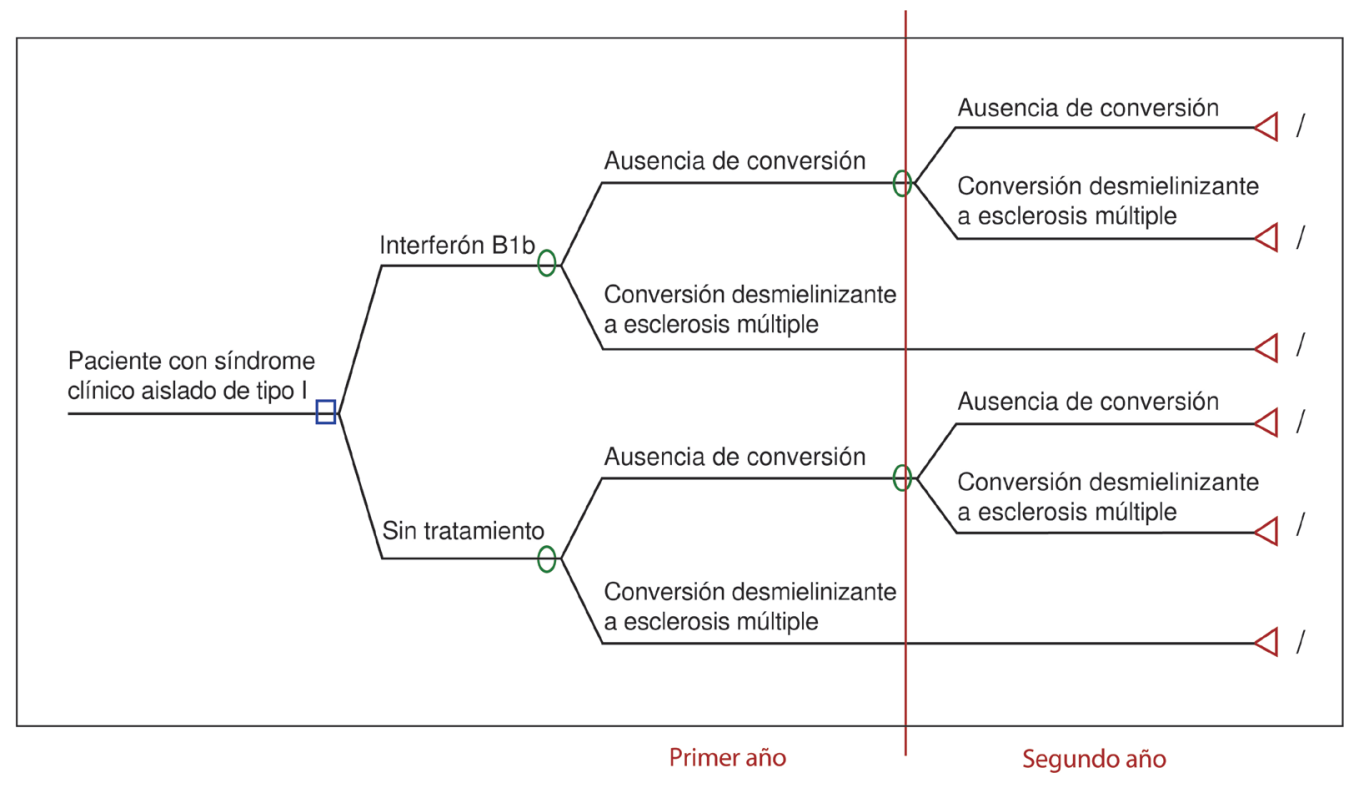

Figura 1. En el árbol de decisiones realizado se analizó la intervención de dar y no dar tratamiento con interferón beta a los pacientes con síndrome clínico aislado. Posteriormente, se evaluaron las probabilidades de presentar o no conversión a esclerosis múltiple en cada grupo durante el primero y el segundo año. 
Cuadro 1. Probabilidad de progresar a esclerosis múltiple en pacientes con síndrome clínico aislado de tipo I con tratamiento y sin él en los primeros dos años según los diferentes estudios publicados

\begin{tabular}{lcccl}
\hline Probabilidad & Valor & Mínimo & Máximo & Fuente \\
\hline Recaída en el primer año con interferón & 0,50 & 0,16 & 0,58 & ETOMS (Lancet 2001) \\
Recaída en el primer año sin tratamiento & 0,68 & 0,27 & 0,78 & BENEFIT (Neurology 2006) \\
Recaída en el segundo año con interferón & 0,55 & 0,33 & 0,67 & CHAMPS (NEJM 2000) \\
Recaída en el segundo año sin tratamiento & 0,86 & 0,44 & 0,88 & Revisión Cochrane 2009 \\
\hline
\end{tabular}

Cuadro 2. La mitad superior del cuadro describe los costos directos del tratamiento con interferón beta por mes, por año y por dos años en Colombia (en dólares). La mitad inferior describe los costos de las recaídas por esclerosis múltiple en Colombia, incluidos los de hospitalización, estudios diagnósticos, tratamiento farmacológico y de rehabilitación y otros.

\begin{tabular}{|c|c|c|c|c|}
\hline & $\begin{array}{l}\text { Costo mensual } \\
\text { interferón (US\$) }\end{array}$ & $\begin{array}{l}\text { Costo primer año } \\
\text { interferón (US\$) }\end{array}$ & $\begin{array}{l}\text { Costo segundo año } \\
\text { interferón (US\$) }\end{array}$ & Fuente \\
\hline Valor & $1.727,66$ & $20.731,93$ & $40.860,02$ & Resolución 0005 de 2011 \\
\hline Mínimo & $1.295,75$ & $15.548,95$ & $30.645,02$ & Ministerio de la Protección Social \\
\hline \multirow[t]{2}{*}{ Máximo } & $2.038,64$ & $24.463,68$ & $48.214,83$ & \\
\hline & $\begin{array}{l}\text { Costo mensual } \\
\text { recaída (US\$) }\end{array}$ & $\begin{array}{l}\text { Costo primer año } \\
\text { recaída (US\$) }\end{array}$ & $\begin{array}{l}\text { Costo segundo año } \\
\text { recaída (US\$) }\end{array}$ & Fuente \\
\hline Valor & $1.258,11$ & $15.097,32$ & $29.754,91$ & Registros Hospital Universitario \\
\hline Mínimo & 871,05 & $10.452,58$ & $20.600,72$ & San Ignacio \\
\hline Máximo & $1.643,89$ & $19.726,68$ & $38.878,80$ & \\
\hline
\end{tabular}

Protección Social de Colombia (33). Los costos de las recaídas por mes y por año se obtuvieron de los análisis realizados por Romero en 2011 sobre el promedio del manejo clínico por mes, de los cuales se tomaron los datos de población colombiana (34).

Se usaron igualmente los datos de los pacientes que consultaron al Hospital Universitario San Ignacio con diagnóstico de esclerosis múltiple - síndrome clínico aislado, a quienes se les aplicó una encuesta para calcular los costos indirectos de la enfermedad. En esta encuesta se incluyeron el número de recaídas, el tratamiento recibido, el número de consultas por urgencias y ambulatorias en un año, el número y el tipo de terapias requeridas, los costos de desplazamiento y los insumos requeridos para deambulación, entre otras variables. Asimismo, se realizó una reunión con expertos en enfermedades desmielinizantes para la revisión de los datos encontrados.

De acuerdo con el árbol de decisiones, se conformaron dos grupos: el primero correspondió a los pacientes con síndrome clínico aislado que recibieron tratamiento con interferón y el segundo a los pacientes con esta enfermedad que no fueron medicados. Posteriormente se tomaron las probabilidades de presentar conversión a esclerosis múltiple definitiva para cada grupo registradas por los estudios CHAMPS, ETOMS, BENEFIT, y se complementaron con una revisión
Cochrane. En el análisis del árbol de decisiones se calcularon las probabilidades a uno y dos años. En el análisis del segundo año se aplicó una tasa de descuento de $3 \%$.

Los costos indirectos de la enfermedad se calcularon con la aplicación de una encuesta a los pacientes con síndrome clínico aislado.

El desenlace primario fue la conversión a esclerosis múltiple clínicamente definitiva. El desenlace del tratamiento fueron los años de vida ajustados por discapacidad (AVAD) ganados y los años de vida ajustados por calidad. Los AVAD corresponden a un indicador que combina el número de años vividos con la calidad de vida experimentada durante esos años. Las utilidades del estado de salud se determinaron con base en las tablas de referencia de Tufts University (35).

Los resultados se analizaron con base en la perspectiva del paciente y la sociedad. La investigación contó con el aval del Comité de Ética Médica del Hospital Universitario San IgnacioPontificia Universidad Javeriana.

\section{Resultados}

Se realizó una búsqueda sistemática de la literatura con el objeto de calcular la probabilidad en uno y dos años de que un paciente con síndrome clínico aislado progrese a esclerosis múltiple definitiva. Se obtuvieron 34 resultados que fueron incluidos en el análisis de efectividad y analizados tomando en 
cuenta si recibían o no el tratamiento. En el cuadro 1 se describen los resultados encontrados en los diferentes estudios analizados.

Según estos resultados, se ha demostrado que el tratamiento con interferón retrasa dos a tres años la conversión a esclerosis múltiple definitiva (2). Se considera que sin tratamiento, la aparición de la enfermedad ocurre en promedio a los 1,7 años. En el estudio BENEFIT, $85 \%$ de los pacientes del grupo placebo cumplía con los criterios diagnósticos luego de dos años del primer evento $(1,2,20,22,36)$.

Con los datos analizados se calculó una probabilidad de progresión a esclerosis múltiple definitiva (recaída) en dos años de 0,50 en el grupo que recibió tratamiento con interferón beta y de 0,85 en el grupo que no recibió tratamiento.

Para calcular los costos directos e indirectos de la enfermedad se recolectaron los datos de los pacientes que consultaron al Hospital Universitario San Ignacio con diagnóstico de síndrome clínico aislado o esclerosis múltiple. Se obtuvo un total de 57 pacientes en la base de datos, de los cuales 94 \% contestó la encuesta.

El costo del interferón para dos años se estimó en US\$ 40.860 ( 1 dólar equivalía en el momento a Col $\$ 1.800)$, es decir, Col $\$ 73^{\prime} 548.000$ y el costo de una recaída o de un síndrome clínico aislado en dos años fue de Col\$ 57'139.200 (US\$ 31.744).

En cuanto a los costos indirectos, se estimó que el síndrome clínico aislado tiene un costo aproximado de Col\$10'958.400 (US\$ 6.088) en dos años. De estos costos, aproximadamente, Col\$ 6'998.400
(US\$ 3.888) correspondieron a incapacidades médicas y Col\$2,340.000 (US\$ 1.300) a los desplazamientos requeridos por los pacientes para su atención médica (cuadro 3). Al realizar el análisis de costo-efectividad, los costos de no dar tratamiento correspondieron a Col\$52'880.400 (US\$ 29.378), mientras que el costo de dar tratamiento fue de Col\$104'846.400 (US\$ 58.248). La diferencia en costos fue de Col\$ 51'964.200 (US\$ 28.869).

En este estudio, los AVAD sin tratamiento fueron de 1,68, mientras que con interferón fueron de 1,74. Por lo tanto, la diferencia en la efectividad 'incremental' (sic.) sólo fue de 0,06 AVAD (cuadro 4). La razón de costo-efectividad 'incremental' (sic.) se calculó en Col\$ 895'417.200 (US\$ 497.454). Además, se consideró un valor equivalente a tres veces el producto interno bruto per cápita, que resultó en US\$20.000 (Col\$36'000.000), como umbral de disposición a pagar en Colombia (37).

Se realizó un análisis de sensibilidad determinístico y probabilístico; para el primer caso, las variables de costo del manejo de la recaída en el segundo año y la efectividad de no dar tratamiento fueron sensibles al modelo, pero ninguna fue determinante en la relación costo-efectividad (figura 2); por otra parte, en la simulación de Monte Carlo con mil pacientes, dar interferón no fue costo-efectivo en la mayoría de los casos, y la nube de puntos se concentró sobre la línea de la disposición a pagar o el umbral de costo-efectividad (figura 3).

\section{Discusión}

Uno de los argumentos que sustentan el inicio temprano del interferón en pacientes con síndrome clínico aislado de alto riesgo es que éste limita

Cuadro 3. Costos indirectos en dólares derivados de pacientes con síndrome clínico aislado en Colombia al mes, al año y a los dos años

\begin{tabular}{lrccc}
\hline $\begin{array}{l}\text { Costos } \\
\text { indirectos (US\$) }\end{array}$ & Mensual & Primer año & Segundo año & Fuente \\
\hline Acompañante & 31 & 377 & 744 & Encuesta aplicada \\
Desplazamientos & 5 & 664 & 1.308 & a pacientes con \\
Incapacidades & 164 & 73 & 3.890 & síndrome clínico \\
Copagos & 6 & 3.087 & 6.087 & aislado de tipo I \\
Total & 200 & & &
\end{tabular}

Cuadro 4. Comparación de costos expresados en dólares y utilidad expresada en AVAD entre dar y no dar interferón a pacientes con síndrome clínico aislado

\begin{tabular}{lcccccc}
\hline Estrategia & Costo (US\$) & $\begin{array}{c}\text { Costo } \\
\text { 'incremental' } \\
\text { (sic.) }\end{array}$ & Efectividad & $\begin{array}{c}\text { Efectividad } \\
\text { 'incremental' } \\
\text { (sic.) }\end{array}$ & $\begin{array}{c}\text { Costo- } \\
\text { efectividad }\end{array}$ & ICER $^{*}$ \\
\hline Sin tratamiento & $29.378,74$ & $\$ 28.869,29$ & 1,685216 & & $\$ 17.433,22$ & \\
Interferón B1b & $58.248,02$ & & 1,74325 & 0,058034 & $\$ 33.413,47$ & $\$ 497.454,71$ \\
\hline
\end{tabular}

* Relación costo-efectividad incremental (sic). 


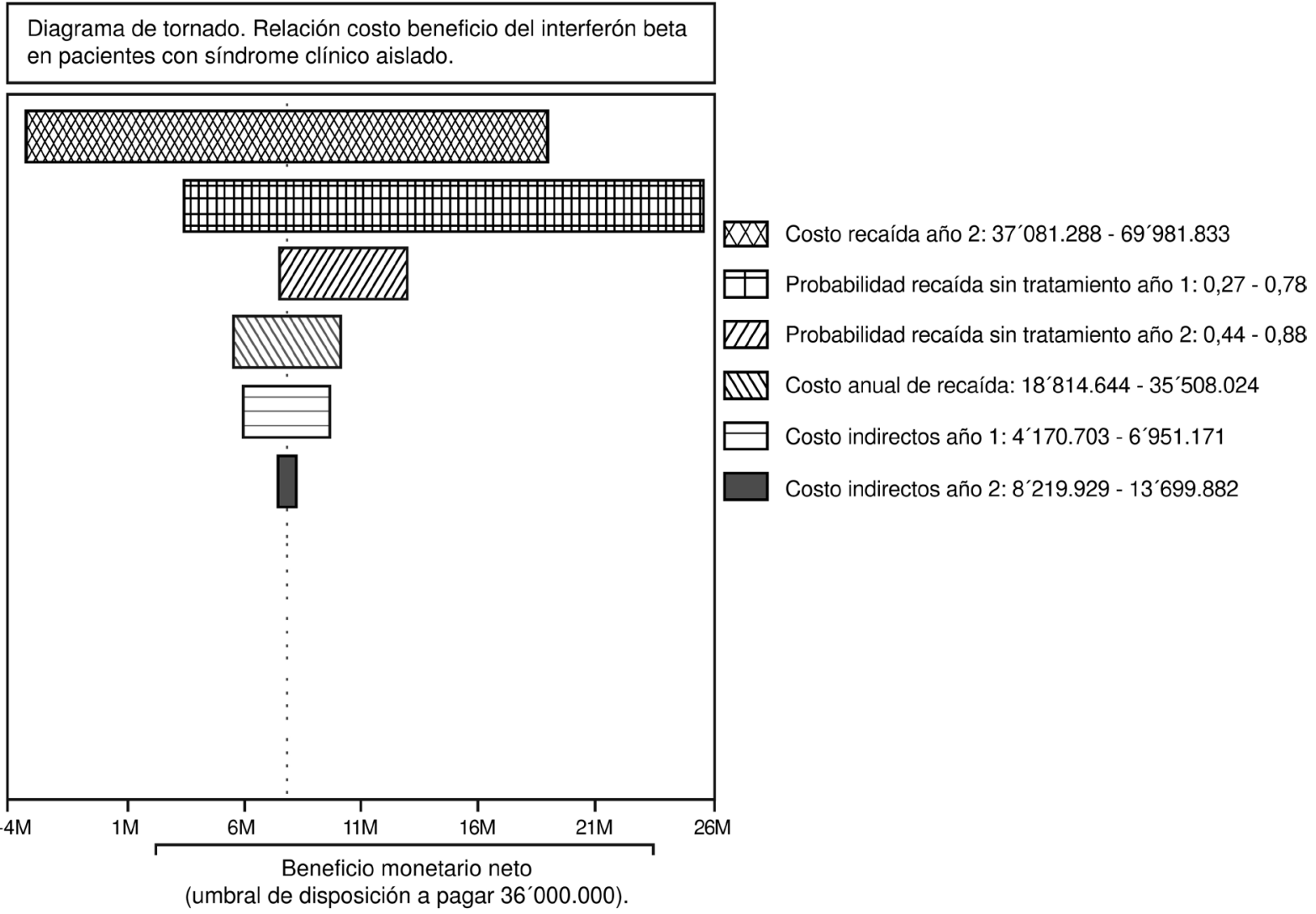

Figura 2. En el diagrama de tornado se observan las variables más sensibles al modelo. El ancho de la barra representa su efecto sobre el resultado final; cuanto más amplia, mayor es el efecto. Con un umbral de disposición a pagar en Colombia de US\$20.000 (Col\$36'000.000), las variables críticas que pueden impactar el beneficio neto monetario fueron el costo de recaída en el segundo año y la probabilidad de recaída sin tratamiento; sin embargo, ninguna en este caso determinó la relación de costo-efectividad 'incremental' (sic.) del tratamiento.

el impacto de la enfermedad en términos de funcionalidad a largo plazo debido a que en los estadios iniciales de la esclerosis múltiple hay una fase inflamatoria con formación de placas, mientras que en etapas más avanzadas el fenómeno que ocurre es la formación de lesiones no inflamatorias, en las cuales hay daño axonal, lesión de oligodendrocitos y activación de la microglía $(1,10,15,19,38,39)$.

Según los resultados obtenidos en el presente estudio, durante los dos primeros años el costo del tratamiento del síndrome clínico aislado con interferón beta sobrepasó los Col\$90’000.000 (US\$ 50.000), cifra en la que cerca de $80 \%$ corresponde a los costos directos del medicamento (aproximadamente, Col\$72'999.000 o US\$40.555), mientras que el costo de la recaída calculado a dos años corresponde a Col\$ 60'778.649 (US\$ 31.744). Los costos indirectos calculados a dos años corresponden a Col\$10'958.400 (US\$ 6.088). Es claro que en el contexto colombiano una ganancia en efectividad de 0,06 AVAD no justifica los altos costos del tratamiento.

En conclusión, la administración del interferón en pacientes con síndrome clínico aislado de alto riesgo no es costo-efectiva en los primeros dos años de la enfermedad.

En cuanto a las limitaciones del estudio, el horizonte temporal de sólo dos años es corto, y es posible que al usar un período más prolongado los resultados puedan cambiar a favor del tratamiento como en otros estudios. Asimismo, las utilidades fueron tomadas de la literatura internacional, pues en Colombia no hay estudios al respecto, lo cual podría incidir un poco en los resultados.

En diversos estudios se ha documentado la efectividad del interferón beta, sin embargo, como se ha demostrado en el presente trabajo, $80 \%$ de los costos directos del tratamiento del síndrome 


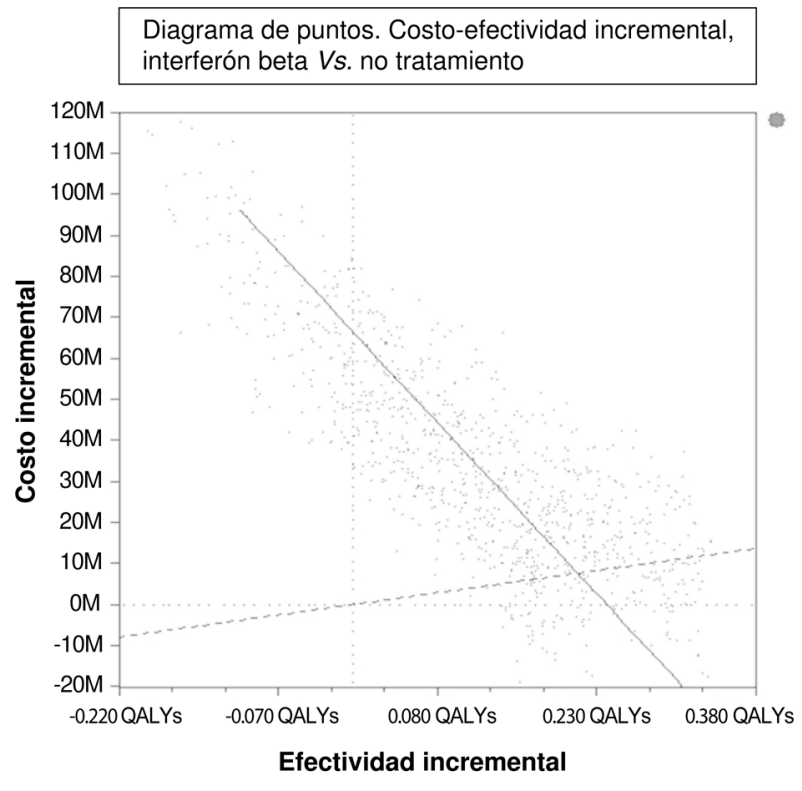

Figura 3. La nube de puntos representa el conjunto de costos y efectividades de acuerdo a una simulación probabilística de mil pacientes; la línea punteada representa la disposición a pagar, por lo tanto, los puntos por encima de ésta indican que el tratamiento evaluado tiende a no ser costo-efectivo.

clínico aislado corresponde al medicamento. Por este motivo, habría que diseñar modelos tomando como base un precio regulado del medicamento y evaluar así su costo-efectividad. Se recomienda, igualmente, hacer nuevos estudios que incluyan una expectativa de vida más amplia.

\section{Conflicto de intereses}

Los autores expresan no tener ningún conflicto de intereses.

\section{Financiación}

La financiación de la investigación provino de recursos personales de los autores.

\section{Referencias}

1. Throwem B. Clinically isolated syndromes. Predicting and delaying multiple sclerosis. Neurology. 2007;68:S12-5. http://dx.doi.org/10.1212/01.wnl.0000277704.56189.85

2. Kappos L, Freedman M, Polman C, Edan G. Effect of early versus delayed interferon beta-1b treatment on disability after a first clinical event suggestive of multiple sclerosis: a 3-year follow-upanalysis of the BENEFIT study. Lancet. 2007;370:38997. http://dx.doi.org/10.1016/S0140-6736(07)61194-5

3. Kappos L, Freedman M, Polman C, Edan G. Subgroups of the BENEFIT study: Risk of developing MS and treatment effect of interferon beta-1b. J Neurol. 2008;255:480-7. http:// dx.doi.org/10.1007/s00415-007-0733-2

4. Pakdaman H, Sahraian M, Fallah A, Pakdaman R, Ghareghozli K. Effect of early interferon beta-1a therapy on conversion to multiple sclerosis in Iranian patients with a first demyelinating event. Acta Neurol Scand. 2007;115:42931. http://dx.doi.org/10.1111/j.1600-0404.2007.00813.x

5. Miller D, Barkhof F, Montalban X, Thompson A, Filippi M. Clinically isolated syndromes suggestive of multiple sclerosis, part I: Natural history, pathogenesis, diagnosis, and prognosis. Lancet Neurol. 2005;4:281-8. http://dx.doi. org/10.1016/S1474-4422(05)70071-5

6. Miller D, Barkhof F, Montalban X, Thompson A, Filippi M. Clinically isolated syndromes suggestive of multiple sclerosis, part 2: Non-conventional MRI, recovery processes, and management. Lancet Neurol. 2005;4:341-8. http://dx.doi.org/10.1016/S1474-4422(05)70095-8

7. Toro J, Sarmiento O, Díaz Del Castillo A, Satizábal CL, Ramírez JD. Prevalence of multiple sclerosis in Bogotá, Colombia. Neuroepidemiology. 2007;28:33-8. http://dx.doi. org/10.1159/000097854

8. Kobelt G, Texier-Richard B. The long-term cost of multiple sclerosis in France and potential changes with diseasemodifying interventions. Mult Scler. 2009;15:741-51. http:// dx.doi.org/10.1177/1352458509102771

9. Iskedjian M, Walker J, Gray T, Vicente C, Einarson T. Economic evaluation of Avonex (interferon beta1a) in patients following a single demyelinating event. Mult Scler. 2005;11:542-51. http://dx.doi.org/10.1191/ $1352458505 \mathrm{~ms} 12110 \mathrm{a}$

10. Kappos L, Polman C, Freedman M, Edan G, Hartung $H$, Miller $D$, et al. Treatment with interferon beta- $1 b$ delays conversion to clinically definite and McDonald MS in patients with clinically isolated syndromes. Neurology. 2006;67:1242-9. http://dx.doi.org/10.1212/01. wnl.0000237641.33768.8d

11. Nielsen M, Moraal B, Polman CH, Poppe P. Classification of patients with a clinically isolated syndrome based on signs and symptoms is supported by magnetic resonance imaging results. Mult Scler. 2007;13:717-21. http://dx.doi. org/10.1177/1352458506074684

12. Swanton J, Fernando K, Dalton CM. Modification of MRI criteria for multiple sclerosis in patients with clinically isolated syndromes. J Neurol Neurosurg Psychiatry. 2006;77:830-3. http://dx.doi.org/10.1136/jnnp.2005.073247

13. Parkin D, Jacoby A, McNamee P. Treatment of multiple sclerosis with interferon B: An appraisal of cost-effectiveness and quality of life. J Neurol Neurosurg Psychiatry. 2000;68:144-49. http://dx.doi.org/10.1136/jnnp.68.2.144

14. Brown M, Kirby S, Kedgel C, Fisk J, Murray T, Bhan V. How effective are disease-modifying drugs in delaying progression in relapsing-onset MS? Neurology. 2007;69:1498-507. http://dx.doi.org/10.1212/01.wnl.0000271884.11129.f3

15. O’Connor P, Kinkel R, Kremenchutzky M. Efficacy of intramuscular interferon beta-1a in patients with clinically isolated syndrome: Analysis of subgroups based on new risk criteria. Mult Scler. 2009;15:728-34. http://dx.doi. org/10.1177/1352458509103173

16. Kappos L, Freedman M, Polman C, Edan G, Hartung $\mathbf{H}$, Miller $\mathbf{D}$, et al. Long-term effect of early treatment with interferon beta- $1 \mathrm{~b}$ after a first clinical event suggestive of multiple sclerosis: 5-year active treatment extension of the phase 3 BENEFIT trial. Lancet Neurol. 2009;8:987-97. http://dx.doi.org/10.1016/S1474-4422(09)70237-6 
17. Jacobs L, Beck R, Simon J, Kinkel P. Intramuscular interferon beta $1 \mathrm{~A}$, therapy initiated during a first demyelinating event in multiple sclerosis. N Engl J Med. 2000;343:898904. http://dx.doi.org/10.1056/NEJM200009283431301

18. Moraal B, PohI C, Polman C, Edan G, Freedman M. Magnetic resonance imaging predictors of conversion to multiple sclerosis in the BENEFIT Study Arch Neurol. 2009;66:134552. http://dx.doi.org/10.1001/archneurol.2009.243

19. Jun-ichi K. MS: Prevention of neural damage by early intervention. Lancet Neurol. 2008;7:3-5. http://dx.doi. org/10.1016/S1474-4422(07)70299-5

20. Goodin D, Bates D. Treatment of early multiple sclerosis: The value of treatment initiation after a first clinical episode. Mult Scler. 2009;15:1175-82. http://dx.doi.org/10. $1177 / 1352458509107007$

21. Comi G, Filippi M, Barkhof, F, Durelli L. Effect of early interferon treatment on conversion to definite multiple sclerosis: A randomized study. Lancet. 2001;357:1576-82. http://dx.doi.org/10.1016/S0140-6736(00)04725-5

22. Jacobs LD, Beck RW, Simon JH. Intramuscular interferon beta-1a therapy initiated during a first demyelinating event in multiple sclerosis; CHAMPS Study Group. N Engl J Med. 2000;343:898-904. http://dx.doi.org/10.1056/ NEJM200009283431301

23. Comi G, De Stefano N, Freedman M, Barkhof F, Polman C, Uitdehaag B, et al. Comparison of two dosing frequencies of subcutaneous beta-1a in patients with a first clinical demyelinating event suggestive of multiple sclerosis (REFLEX): A phase 3 randomized controlled trial. Lancet Neurol. 2001;10:33-41. http://dx.doi.org/10.1016/S14744422(11)70262-9

24. Bermel R, Rudick R. Interferon treatment for multiple sclerosis. Neurotherapeutics. 2007;4:633-46.

25. Prosser L, Kuntz K, Bar-Or A, Weinstein M. Costeffectiveness of interferon beta-1a, interferon beta- $1 b$, and glatiramer acetate in newly diagnosed non-primary progressive multiple sclerosis. Value Health. 2004;7:55468. http://dx.doi.org/10.1111/j.1524-4733.2004.75007.x

26. Flachenecker P, Rieckmann P. Early intervention in multiple sclerosis better outcomes for patients and society? Drugs. 2003;63:1525-33. http://dx.doi.org/10.2165/00003495200363150-00001

27. Coyle P, Arnason B, Hurwitz B, Lublin F. Optimizing outcomes in multiple sclerosis - a consensus initiative. Mult Scler. 2008;14:S5-35. http://dx.doi.org/10.1177/ 1352458508101134

28. Lazzaro C, Bianchi C, Peracino L, Zacchetti P, Uccelli E. Economic evaluation of treating clinically isolated syndrome and subsequent multiple sclerosis with interferon $b-1 b$. Neurol Sci. 2009;30:21-31. http://dx.doi.org/10.1007/s10072009-0015-0
29. Nuijten C, Hutton J. Cost-effectiveness analysis of interferon beta in multiple sclerosis: A Markov process analysis. Value Health. 2002;5:44-54. http://dx.doi.org/10.1046/j.1524-4733. 2002.51052.x

30. Kobelt G, Berg J, Atherly D, Hadjimichael O. Costs and quality of life in multiple sclerosis. A cross-sectional study in the United States. Neurology. 2006;66:1696-702. http:// dx.doi.org/10.1212/01.wnl.0000218309.01322.5c

31. Phillips C. The cost of multiple sclerosis and the cost effectiveness of disease-modifying agents in its treatment. CNS Drugs. 2004;18:561-74. http://dx.doi.org/ 10.2165/00023210-200418090-00002

32. Kendrick $\mathbf{M}$, Johnson $\mathbf{K}$. Long term treatment of multiple sclerosis with interferon- $\beta$ may be cost effective. Pharmacoeconomics. 2000;18:45-53. http://dx.doi.org/10. 2165/00019053-200018010-00005

33. Ministerio de la Protección Social. Resolución 0005 de 2011. Valor máximo de recobro de unidad, tratamiento mes Interferón Beta 1b. Fecha de consulta: 28 de septiembre de 2013. Disponible en: http://www.med-informatica.com/OBSERVAMED/ Deposito_legal/MPS_Resolucion005de11ene2011_ ModifRes5229ValorMaxReco20medicamentos.pdf.

34. Romero M, Arango C, Alvis N. Costos de la esclerosis múltiple en Colombia. Value Health. 2011;14:S48-50. http:// dx.doi.org/10.1016/j.jval.2011.05.023

35. Tufts Medical Center. Cost-Effectiveness Analysis Registry (CEA). Fecha de consulta: 10 de octubre del 2011. Disponible en: http://research.tufts-nemc.org/cear/ default.aspx.

36. Clerico M, Faggiano $\mathbf{F}$, Palace J, Rice G, Tintorè M, Durelli L. Recombinant interferon beta or glatiramer acetate for delaying conversion of the first demyelinating event to multiple sclerosis. Cochrane Database Syst Rev. 2008;16:CD005278. http://dx.doi.org/10.1002/14651858. CD005278.pub3

37. Ministerio de la Protección Social. Producto interno bruto e inflación en Colombia. Fecha de consulta: 10 de octubre del 2011. Disponible en: www.miniprotecciónsocial.gov.co/ estadisticas/Estadisticas/evolucion del pib e inflacion en colombia, 2000-2009.pdf.

38. Confavreux C, Vukusic S. Natural history of multiple sclerosis: A unifying concept. Brain. 2006;129:606-16. http:// dx.doi.org/10.1093/brain/awl007

39. Cottrell D, Rice G. The natural history of multiple sclerosis: A geographically based study. Brain. 1999;122:1941-9. http://dx.doi.org/10.1093/brain/112.1.133 